\section{Gravity measured with record precision}

\section{The gravitational constant, $G$, which governs the strength of gravitational interactions, is hard to measure accurately. Two independent determinations of $G$ have been made that have the smallest uncertainties So far. SEE ARTICLE P.582}

\section{STEPHAN SCHLAMMINGER}

A lthough gravity seems strong in our everyday lives, such as when lifting a heavy object, it is the weakest of the four fundamental forces. The gravitational force between two bodies is proportional to the masses of these bodies. If one of the bodies is Earth, the force can be considerable. But if the bodies are objects in a laboratory, the force can be too small to measure accurately. For example, the gravitational force between two 1-kilogram objects separated by 1 metre is equivalent to the weight of a few biological cells. For this reason, the gravitational constant, $G$, which quantifies the strength of this force, is one of the most poorly defined physical constants. But on page 582, Li et al. ${ }^{1}$ report high-precision measurements of $G$ using two different techniques.

In 1798, the scientist Henry Cavendish determined $G$ for the first time in the laboratory, using an instrument called a torsion balance ${ }^{2}$. In Cavendish's work, the torsion balance consisted of a dumb-bell that was suspended from its centre by a thin fibre. A gravitational force was applied to the masses at the ends of the dumb-bell, acting perpendicularly to the bar of the dumb-bell and to the axis of the fibre. This force led to a rotation of the dumb-bell about this axis, causing the fibre to twist.

Eventually, the dumb-bell reached a position at which the twisting force of the fibre balanced the gravitational force. The rotation angle of the dumb-bell in this position was recorded. The gravitational force was then applied in the opposite direction and a second rotation angle was measured. The magnitude of the gravitational force was calculated from the difference between these two angles.

In torsion-balance experiments, the gravitational force is provided by a well-characterized assembly of external masses. These masses are

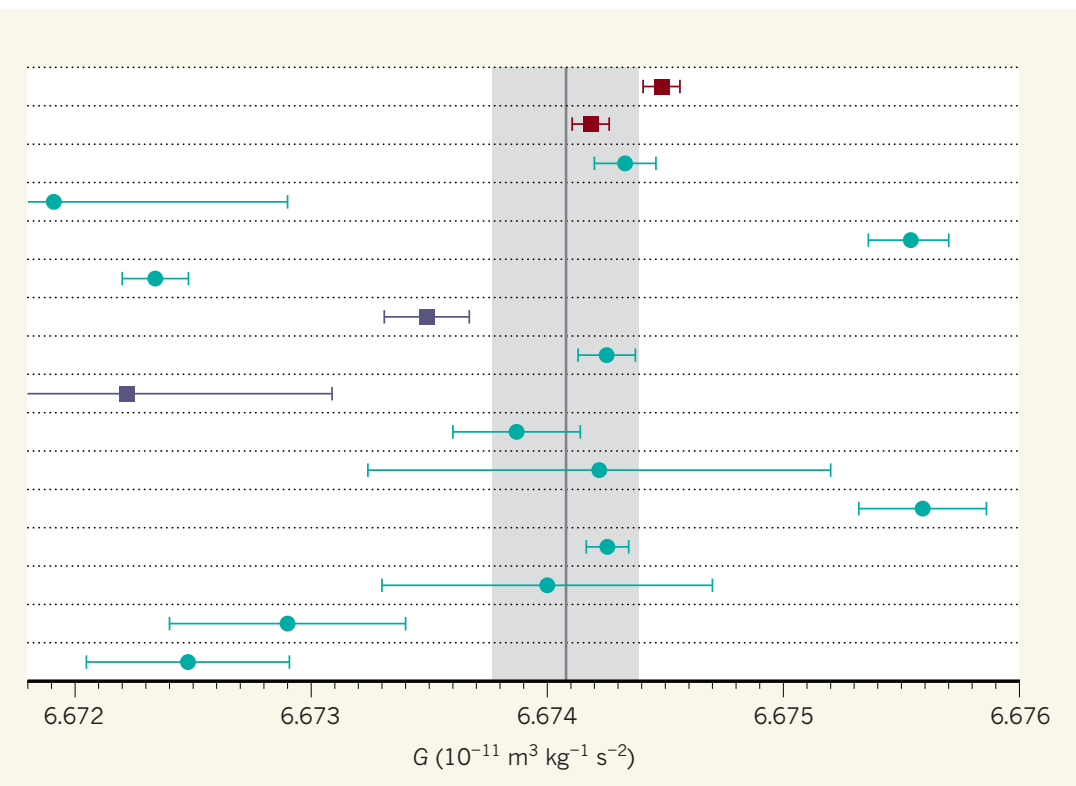

Figure 1 | Measurements of the gravitational constant. The strength of the gravitational force between two bodies is described by the gravitational constant, $G$, which can be expressed in units of cubic metres per kilogram per square second. The data points are high-precision measurements of $G$ taken over the past 40 years, with uncertainties indicated by the error bars. The points marked by squares are results obtained by Li et al. in current work ${ }^{1}$ (red) and in previous work ${ }^{8,9}$ (purple). The vertical grey line denotes the value of $G$ adopted by the Committee on Data for Science and Technology, with an uncertainty indicated by the shaded area ${ }^{11}$. (Adapted from Fig. 3 of ref. 1.) moved between two or more different positions to change the direction and magnitude of the force. Because the dumb-bell rotates in a horizontal plane, the otherwise overwhelming effects of Earth's gravity on the experiments are negligible. Over the years, many techniques have been developed to measure $G$ using a torsion balance ${ }^{3}$. In 2000, a substantial improvement in the precision of these experiments was achieved by replacing the dumb-bell with a thin plate $^{4}$ (also termed a plate pendulum).

$\mathrm{Li}$ and colleagues built two plate-containing torsion balances that are based on different measurement techniques: the time-of-swing (TOS) method $^{5}$ and the angular-accelerationfeedback (AAF) method $^{6}$ (see Fig. 1 of the paper $^{1}$ ). In the TOS method, the rotation of the plate is oscillatory. $G$ is calculated from the change in the speed of the oscillation when the external masses are in two different configurations. By contrast, in the AAF method, two turntables are used to rotate the torsion balance and the external masses individually. $G$ is determined from the angular acceleration of the turntable associated with the torsion balance when the amount of twisting of the fibre is reduced to zero.

The authors obtained $G$ values of $6.674184 \times 10^{-11}$ and $6.674484 \times 10^{-11}$ cubic metres per kilogram per square second for the TOS method and the AAF method, respectively. The relative uncertainties are the smallest reported so far: about 11.6 parts per million. By comparison, the previous record, which was achieved using the AAF method, was 13.7 parts per million ${ }^{4}$.

Li et al. carried out their experiments with great care and gave a detailed description of their work. The study is an example of excellent craftsmanship in precision measurements. However, the true value of $G$ remains unclear. Various determinations of $G$ that have been made over the past 40 years have a wide spread of values (Fig. 1). Although some of the individual relative uncertainties are of the order of 10 parts per million, the difference between the smallest and largest values is about 500 parts per million.

There are at least two possible explanations for this discrepancy. One is that the technical details of one or more of the experiments were not fully understood, which could have led either to a systematic shift in the reported values of $G$ or to uncertainties that were not included in the reported uncertainties of $G$. An example of the former is the effect of a fibre property, called anelasticity, that could bias the TOS method - an effect that was first pointed out in 1995. A second possibility is that some unknown physics could explain the scatter in the published values. Although this possibility is, of course, the more exciting, it is also the less likely. Nevertheless, it should not be dismissed lightly.

At this point, it is as important to try to understand the discrepancy between the different results as it is to make new 
measurements. Even Li and colleagues' results are in disagreement: the values of $G$ determined in the two current experiments, as well as values obtained in two previous experiments at the same laboratory ${ }^{8,9}$, are statistically inconsistent with one another. The authors speculate that fibre anelasticity might be responsible, but they do not give a definitive explanation.

Because all four of these experiments were carried out at the same institution, it should be more straightforward to compare them than it would be to compare different experiments from various groups around the globe. An excellent opportunity exists, therefore, to uncover the causes of the discrepancy and, in turn, to learn more about the true value of $G$. Li et al. should be encouraged to take on this challenge. In the end, if we want to understand the measurements of $G$, we must find the reasons for the inconsistent results ${ }^{10}$.
Stephan Schlamminger is in the Fundamental Electrical Measurements Group, National Institute of Standards and Technology, Gaithersburg, Maryland 20899, USA.

e-mail: stephan.schlamminger@nist.gov

1. Li, Q. et al. Nature $560,582-588$ (2018)

2. Cavendish, H. Phil. Trans. R. Soc. B 88, 469-526 (1798).

3. Rothleitner, C. \& Schlamminger, S. Rev. Sci. Instrum. 88, 111101 (2017).

4. Gundlach, J. H. \& Merkowitz, S. M. Phys. Rev. Lett. 85, 2869-2872 (2000).

5. Reich, F. Abh. Math.-Phys. Cl. Königliche Sächsischen Ges. Wiss. 1, 384-430 (1852)

6. Rose, R. D., Parker, H. M., Lowry, R. A., Kuhlthau, A. R. \& Beams, J. W. Phys. Rev. Lett. 23, 655-658 (1969).

7. Kuroda, K. Phys. Rev. Lett. 75, 2796 (1995).

8. Hu, Z. K., Guo, J. O. \& Luo, J. Phys. Rev. D 71 , 127505 (2005)

9. Tu, L. C. et al. Phys. Rev. D 82, 022001 (2010).

10. Quinn, T. Nature 505, 455 (2014).

11.Mohr, P. J., Taylor, B. N. \& Newell, D. B. Rev. Mod. Phys. 88, 035009 (2016)

\section{A new green revolution on the horizon}

\section{Manipulation of the transcription factor OsGRF4 can improve the efficiency with which some high-yielding cereal crops use nitrogen. This discovery has implications for sustainable agriculture. SEE ARTICLE P.595}

\section{FANMIAO WANG \& MAKOTO MATSUOKA}

$\mathrm{T}$ The green revolution of the mid-twentieth century saw the development of highyielding varieties of rice and wheat for use in agriculture. But to produce high yields, these green-revolution varieties require a large supply of nitrogen. Developing greenrevolution varieties that use nitrogen more efficiently is an important goal for sustainable crop breeding. On page 595, Li et al. ${ }^{1}$ report a previously unknown function for the rice transcription factor OsGRF4 in nitrogen use. By modulating the OsGRF4 gene, the researchers produced plants that use nitrogen efficiently and have a high yield.

Proteins of the DELLA family inhibit plant growth, whereas hormones called gibberellins promote plant growth by triggering the destruction of DELLA proteins. Green-revolution varieties of rice and wheat harbour genetic mutations that lead to the accumulation of DELLA proteins. As a result, these plants are shorter than are normal varieties, and so are resistant to lodging ${ }^{2,3}$ - the process by which plants are flattened by wind and rain. This lodging resistance is a fundamental mechanism for achieving increased crop yield in green-revolution varieties.

DELLA accumulation also inhibits nitrogen uptake and nitrogen-related growth responses - traits that are associated with the inefficient use of nitrogen ${ }^{4}$. Consequently, farmers have to apply large amounts of environmentally damaging nitrogen-based fertilizer to their crops to achieve high yields in green-revolution varieties. Although DELLA accumulation increases the yield, it therefore also has a negative impact in terms of sustainable agriculture.

Li et al. set out to overcome the negative impact of DELLA accumulation. They crossed varieties of the rice subspecies Oryza sativa indica that showed differing rates of nitrogen uptake. They then performed genetic analyses on the resulting plants, which had a range of yields. In doing so, they found that OsGRF4 is associated with nitrogen uptake. OsGRF4 has previously been found to regulate the size of rice grains ${ }^{5-7}$ and the levels of growth molecules called cytokinins ${ }^{8}$, both of which affect crop yield. But no relationship between OsGRF4 and nitrogen-use efficiency has previously been described.

The researchers genetically engineered green-revolution varieties of rice to lack OsGRF4. Compared with control plants carrying the wild-type gene, mutants showed less nitrogen-dependent growth and reduced nitrogen uptake and assimilation (the process by which inorganic nitrogen from fertilizers is converted into useful organic compounds such

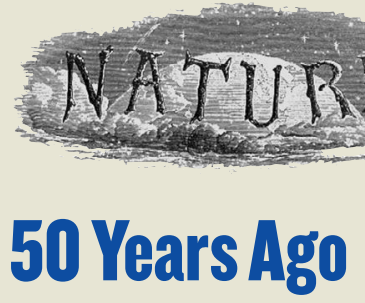

Mr J. H. Brazell of the Meteorological Office has compiled a book of weather statistics for the London area which promises to become a well-thumbed reference ... The year $1841 \ldots$ is the first year for which regular official meteorological observations are available ... Mr Brazell has taken this opportunity to delve into earlier chronicles to find what London's weather was like before 1841 ... A rare feature of London's climate has been the freezing of the Thames ... During twenty-three winters between 1260 and 1814 , the ice on the river was thick enough to allow pedestrians to cross from one bank to the other. It became the custom for frost fairs to be held on the frozen Thames, starting from small beginnings in the winter of 1309-10 when people danced around a bonfire built on the ice, to the great frost fairs of the 17 th, 18th and 19th centuries, when the frozen river supported streets of shops and booths.

From Nature 31 August 1968

100 Years Ago

The July issue of Science Progress contains an interesting article by Sir Henry Thompson on the food requirements of a normal workingclass family. A comparison is instituted between the physiological values of the diets reported upon by the Board of Trade in pre-war times and some data collected by the War Emergency Committee in 1917 ... Sir Henry Thompson has employed a more liberal scale of requirements for children than the older standard of Atwater, which is now generally recognised to be unsatisfactory. The three diets do not differ greatly in respect of energy-value; the highest average is that of the urban working-class families (1913), yielding 3410 calories; the lowest, the 1917 sample, is 3160 calories, a reduction of but 250 calories.

From Nature 29 August 1918 